\title{
TNF and receptors in organ-specific autoimmune disease: multi-layered functioning mirrored in animal models
}

\author{
George Kassiotis and George Kollias \\ Institute for Immunology, Biomedical Sciences Research Center “Alexander Fleming,” Vari, Greece \\ Address correspondence to: George Kollias, Institute for Immunology, Biomedical Sciences Research Center "Alexander Fleming," \\ PO Box 74145, Varkiza 166 02, Greece. Phone: (301) 965-6507; Fax: (301) 965-6563; E-mail: giorgos_kollias@hol.gr. \\ George Kassiotis's present address is: Division of Immunology, National Institute for Medical Research, Mill Hill, London, United Kingdom.
}

Inhibition of TNF in rheumatoid arthritis $(\mathrm{RA})$ is proving efficacious for a large number of patients (1). Despite this exciting outcome, however, there is still little knowledge of the actual biology that is targeted by this therapy. Potential effector activities of TNF that may be relevant to the observed therapeutic impact are numerous and include its well-known proinflammatory activities, the potential activating effects it may have on important cell types (e.g., macrophages or synoviocytes), and its functions in shaping innate and adaptive immunity (2). TNF is usually overproduced in chronic inflammatory/autoimmune diseases, such as RA and multiple sclerosis (MS), and this overproduction leads to strikingly similar pathologies when reproduced in transgenic animal models (see ref. 2 and references therein). Moreover, pharmacological inhibition of TNF in collagen-induced arthritis (CIA) and experimental autoimmune encephalomyelitis (EAE), autoimmune animal models for RA and MS, respectively, has yielded encouraging results. These notions led the way to the use of anti-TNF therapies in the clinic. Surprisingly, however, systemic blockade of TNF in MS patients led to immune activation and increased disease activity $(3,4)$. Further insight into the mechanisms of TNF function is therefore required, and more work with the animal models is necessary.

\section{The role of TNF in the induction of autoimmune disease}

In this issue of the JCI, Campbell and colleagues revisit the role TNF may play in the induction of autoimmune arthritis (5). They show that following immunization with type II collagen, TNF-deficient mice develop severe inflammatory arthritis and, interestingly, lymphadenopathy as well. Although the two phenotypes appear unrelated, they provide addition- al insights into the potential function of TNF in autoimmunity and autoimmune disease. The finding that TNF is not an absolute requirement for the induction of organ-specific autoimmune disease is not unprecedented. In earlier studies, p55 TNF receptor-deficient (p55 TNFR-deficient) mice were found to develop CIA with a low incidence and in a milder form (6). Similarly, TNF-deficient mice are not protected against EAE, although onset of disease is consistently delayed (ref. 2 and references therein). It appears, therefore, that TNF is generally dispensable in the induction of organ-specific autoimmune disease, although it clearly contributes. The theme that emerges is that TNF is important (and therefore anti-TNF therapy would be effective) only during certain phases of disease induction. Such indications from the CIA model may also provide clues as to why a good percentage of the human RA patients receiving anti-TNF treatments show limited or no response to the drug.

Other properties of TNF, such as regulation of antibody responses (7), may also be important in the CIA model. Collagen-specific humoral and cellular responses are perhaps the most relevant factors in CIA, and interestingly, Campbell and his colleagues demonstrate that inability of TNF-deficient mice to sustain the anti-collagen IgG response correlates well with the overall reduced disease in these mice (5). The arthritogenic mechanisms operating in the absence of TNF in the CIA model (or in the nonresponding patients) are not completely understood and should be targeted in future investigations.

\section{Dual and opposing roles for TNF in chronic autoimmune disease}

The data by Campbell et al. (5) also add to the emerging concept that TNF may not only be dispensable for the progres- sion of disease caused by an organ-specific $T$ cell response, but that it may actually inhibit it. An immune-suppressive role for TNF has been proposed in several models of systemic (ref. 8 and references therein) and organ-specific autoimmune diseases $(8,9)$. A dual role for TNF in EAE has recently being revealed directly in TNF-deficient mice (10). Despite being clearly proinflammatory during disease initiation, TNF exhibited potent immune- and disease-suppressive properties at later stages of the disease, which provided also an explanation for the disease-aggravating outcome of anti-TNF therapy of MS. Interestingly, in the study by Campbell et al. (5), collagen-specific $T$ cells from TNF-deficient mice retained their proliferative capacity in vitro and produced significantly higher amounts of IFN- $\gamma$. Enhanced IFN- $\gamma$ production in TNF-deficient mice could indicate a lack of collagen-specific $\mathrm{T}$ cell regulation or altered polarization. However, joint disease at the time point studied, 60 days after immunization, was milder in TNFdeficient mice. It is therefore important to analyze in detail the effect of TNF deficiency on the collagen-specific $T$ cell response and to determine the course of disease in these mice for prolonged periods of time, which would more accurately reflect the chronicity of human RA.

\section{Potential advantages of anti-p55 TNFR therapies}

Enhanced and prolonged $T$ cell reactivity in TNF-deficient mice may be explained in several ways. One possibility is that the absence of TNF signaling interferes with apoptosis of activated $\mathrm{T}$ cells. Indeed, $\mathrm{T}$ cell apoptosis is impaired in mice lacking the p55 TNFR (11), perhaps accounting for the lymphadenopathy reported by Campbell et al. (5) and the observed accumulation of $\mathrm{T}$ cells with an activated/memory 
phenotype (CD44hi CD45RB ${ }^{\text {lo }} \mathrm{CD}^{+}$). In fact, increased numbers of these $T$ cells can be found even in unmanipulated TNF- or p55 TNFR-deficient mice $(10,12)$, probably reflecting a response to environmental antigens an effect that may therefore vary depending on housing conditions. These numbers increase upon immunization, although we have not observed overt lymphadenopathy in TNF-deficient mice immunized with myelin antigens. In any case, these accumulating $\mathrm{T}$ cells include many mycobacteria-specific $\mathrm{T}$ cells and should not be directly linked to autoimmune disease progression. In the study by Campbell et al. (5), there was no correlation between arthritis and lymphoproliferative disease in TNF-deficient mice. Impaired p55 TNFR-dependent $\mathrm{T}$ cell apoptosis may contribute to a general immune-suppressive function of TNF, but it does not seem to be directly pathogenic. Furthermore, despite impaired $\mathrm{T}$ cell apoptosis, mice lacking the p55 TNFR do not exhibit enhanced self-reactivity to the extent seen in TNF or double p55/p75 TNFR-deficient mice, nor do they develop autoimmune disease (10). Therefore, lack of p55 TNFR-mediated apoptosis cannot fully account for the abnormally enhanced pathogenic selfreactivity in TNF-deficient mice (10).
Together these findings suggest that TNF exerts additional immune-suppressive properties independently of the p55 TNFR. If so, given that this receptor mediates most, if not all, of the proinflammatory activities of TNF in EAE (10, 13) and CIA (6), these findings may reveal an unforeseen opportunity for immune intervention. Conceivably, blocking the p55 TNFR instead of TNF in organ-specific autoimmunity may prove advantageous, since it may inhibit the deleterious proinflammatory and tissue damaging activities of TNF without compromising its immune-suppressive properties. Therefore, whether the p55 TNFR-independent immune-suppressive function of TNF on myelin-reactivity (10) also operates in collagen-reactivity or in other organ-specific autoimmune conditions emerges as a pressing question whose answer could inspire a fundamental change in the design of TNF/TNFR modulating therapies for human autoimmune disease.

1. Feldmann, M., and Maini, R.N. 2001. Anti-TNF$\alpha$ therapy of rheumatoid arthritis: what have we learned? Annu. Rev. Immunol. 19:163-196.

2. Kollias, G., Douni, E., Kassiotis, G., and Kontoyiannis, D. 1999. On the role of tumor necrosis factor and receptors in models of multiorgan failure, rheumatoid arthritis, multiple sclerosis and inflammatory bowel disease. Immunol. Rev. 169:175-194.

3. van Oosten, B.W., et al. 1996. Increased MRI activity and immune activation in two multiple sclerosis patients treated with the monoclonal anti-tumor necrosis factor antibodycA2. Neurology. 47:1531-1534.
4. 1999. TNF neutralization in MS: results of a randomized, placebo-controlled multicenter study. The Lenercept Multiple Sclerosis Study Group and The University of British Columbia MS/MRI Analysis Group. Neurology. 53:457-465.

5. Campell, I.K., O'Donnell, K., Lawlor, K.E, and Wicks, I.P. 2001. Severe inflammatory arthritis and lymphadenopathy in the absence of TNF. J. Clin. Invest. 107:1519-1527.

6. Mori, L., Iselin, S., De Libero, G., and Lesslauer, W. 1996. Attenuation of collagen-induced arthritis in 55-kDa TNF receptor type 1 (TNFR1)-IgG1treated and TNFR1-deficient mice. J. Immunol. 157:3178-3182.

7. Pasparakis, M., Alexopoulou, L., Episkopou, V., and Kollias, G. 1996. Immune and inflammatory responses in TNF alpha-deficient mice: a critical requirement for TNF alpha in the formation of primary B cell follicles, follicular dendritic cell networks and germinal centers, and in the maturation of the humoral immune response. J. Exp. Med. 184:1397-1411.

8. Cope, A.P. 1998. Regulation of autoimmunity by proinflammatory cytokines. Curr. Opin. Immunol. 10:669-676.

9. Grewal, I.S., et al. 1996. Local expression of transgene encoded TNF alpha in islets prevents autoimmune diabetes in nonobese diabetic (NOD) mice by preventing the development of auto-reactive islet-specific T cells. J. Exp. Med. 184:1963-1974.

10. Kassiotis, G., and Kollias, G. 2001. Uncoupling the proinflammatory from the immunosuppressive properties of tumor necrosis factor (TNF) at the p55 TNF receptor level: implications for pathogenesis and therapy of autoimmune demyelination. J. Exp. Med. 193:427-434.

11. Lenardo, M., et al. 1999. Mature T lymphocyte apoptosis-immune regulation in a dynamic and unpredictable antigenic environment. Annu. Rev. Immunol. 17:221-253.

12. Hill, G.R., et al. 2000. The p55 TNF-alpha receptor plays a critical role in T cell alloreactivity. $J$. Immunol. 164:656-663.

13. Eugster, H.P., et al. 1999. Severity of symptoms and demyelination in MOG-induced EAE on TNFR1. Eur. J. Immunol. 29:626-632. 